\title{
Nirschl tennis elbow release with or without drilling
}

\author{
A Khashaba
}

\begin{abstract}
Nirschl release appears to be a very successful technique for surgically suitable cases of tennis elbow. However, although the drilling or decortication aspect of the procedure was thought to be of benefit to the immediate outcome, this has not actually been confirmed. This randomised double blind comparative prospective trial shows that drilling confers no benefit and actually causes more pain, stiffness, and wound bleeding than not drilling.

(Br F Sports Med 2001;35:200-201)
\end{abstract}

Keywords: Nirschl release; lateral epicondyle; tennis elbow; elbow; epicondylitis

The main presenting complaint of tennis elbow is localised pain over the lateral epicondyle, particularly during activities that require active wrist extension power. As a corollary to this, patients also complain of inability to lift objects or make powerful flexing movements of the wrist because this also painfully stretches the extensor muscles.

A variety of surgical techniques are currently available and routinely used for the treatment of tennis elbow. Nirschl's technique ${ }^{1}$ involves (a) excision of the histologically proven degenerate fibroblastic origin of the extensor carpi radialis brevis (with or without the digitorum communis if affected) from the lateral epicondyle, and $(b)$ decorticating or making drill holes (three of $2 \mathrm{~mm}$ diameter) through the near cortex of the anterolateral lateral humeral condyle in the hope of increasing the blood supply to the degenerate area to improve healing and hopefully outcome. Nirschl release (including drilling) is $85 \%$ successful, ${ }^{1}$ which is comparable to published results for simple extensor origin release ${ }^{23}$; however, simple release in our unit conferred only about $50 \%$ success.

Orthopaedic Unit, West Wales Hospital, Camarthen, Wales, UK A Khashaba

Correspondence to: Mr Khashaba, 2 Colwyn Avenue, Perivale, Middlesex UB6 8JX, UK

ahmedkhashaba@hotmail.com

Accepted 25 January 2001 drilling was performed or not. Patients also attended the clinic after two weeks for wound inspection and removal of sutures (skin staples). All patients were operated on as day cases by the same surgeon (the author).

To make the sample study relevant to the general population, this study included all patients (male/female) presenting to their general practitioners. People less than 18 years old rarely suffer from this complaint and were excluded from the study. In this particular sample, there were no revision tennis elbows; all were primary cases. All patients were otherwise fit (American Society of Anesthesiologists category I (ASA-I)).

In this study, treatment protocols in our unit for tennis elbow involved: (a) resting the affected elbow from aggravating causes and a standard course of physiotherapy; if this failed, (b) an injection of local anaesthetic (bupivicaine $0.5 \%$ ) and steroid (40 $\mathrm{mg}$ depo-medrone) into the area of tenderness. If the injection provided more than six months of symptomatic relief, the patient was permitted to have another injection. If the injection worked temporarily, but for less than six months, further injections were found to be less successful or not helpful at all, and these patients were deemed suitable for surgery (sooner rather than later $^{2}$ ) and were included in this trial. If the injection did not work at all, the patients were probably not suffering from tennis elbow and would not be suitable for surgery.

Advice from several statistics departments and the paper by Russell ${ }^{4}$ indicated that data on 18 or more elbows would be required to provide statistical significance, assuming a significance level and therefore type I error $(\alpha)$ of 0.05 and power $(1-\beta)$ of $80 \%$ - that is, type II error $(\beta)$ of $20 \%$ for a two tailed Student's $t$ test. A total of 23 elbows (18 patients) were operated on. The null hypothesis was that there is no difference in outcome between drilling and not drilling - that is, there is only a 5\% chance of rejecting the null hypothesis when it is actually true. It was also assumed that one category on the visual analogue pain score was clinically significant.

Outcome was assessed in two ways. Subjectively, by asking the patient to mark on a Likud visual analogue pain scale before and three and six months after surgery the worst pain experienced during normal activities of daily living. On the $10 \mathrm{~cm}$ scale, zero was equal to no pain at all, and $10 \mathrm{~cm}$ was equivalent to the worst imaginable pain. As a further point of interest, each time that the patients were asked to record their postoperative pain, they were asked to rerecord on the same scale their remembered preoperative pain score. Secondly, and objectively, the patients' wrist extension strength was 
physically quantified (which has never been done before as far as I am aware) using an originally designed AK-7000 extensionometer spring balance construct, the handle of which was designed to force the wrist to use mainly the extensor muscles on the radial side of the wrist, which are those primarily affected by tennis elbow. A series of three wrist power extensions were recorded to obtain an average for that wrist; the contralateral wrist power was also recorded.

\section{Results}

At three and six months after the operation, outcomes were assessed for all 23 elbows (18 patients) operated on. A median outcome for a single elbow was as follows: preoperative pain $6.8 \mathrm{~cm}$; preoperative average power $2.3 \mathrm{~kg}$; average power of unaffected elbow $11 \mathrm{~kg}$; at three months after the operation, remembered preoperative pain $8.9 \mathrm{~cm}$; postoperative pain $3.5 \mathrm{~cm}$; postoperative pain difference $8.9-3.5$ $=5.4 \mathrm{~cm}$; postoperative average power $6.1 \mathrm{~kg}$; at six months after the operation, remembered preoperative pain $7.8 \mathrm{~cm}$; postoperative pain $0.5 \mathrm{~cm}$; postoperative pain difference $7.8-0.5$ $=7.3 \mathrm{~cm}$; postoperative average power $10.1 \mathrm{~kg}$.

On the basis of the above example, the average collated postoperative pain score differences for the whole trial, combining those at three and six months, are: drilled, $4.6 \mathrm{~cm}$ improvement in pain; not drilled, $6.8 \mathrm{~cm}$ improvement in pain.

The average power improvement at six months was $+6.5 \mathrm{~kg}$ for non-drilled cases and $+5.2 \mathrm{~kg}$ for drilled cases.

\section{Discussion}

From this median example it can be seen that the affected elbow is weaker during an extension curl than the unaffected elbow. It is apparent that patients also tend to forget or re-evaluate their perceived preoperative pain level after their operation. Typically patients who benefit considerably from their operation tend to overrate their remembered preoperative pain level. When assessing the results, one needs to compare like with like, therefore the patient's remembered preoperative pain score was used, as this was on the same scoring scale level as their postoperative scores.
On average, postoperative pain decreased on the analogue score and further improved at six months, with most patients going back to work at three months (sooner for non-manual workers, whose jobs did not require a lot of muscular power). Most of the elbows (87\%) affected were on the dominant side. Occupations of the patients included nurses, gardeners, forresters, and housewives.

Average extension power improved after the operation but did not quite reach the power level of the unaffected elbow at six months, except in male patients involved in heavy manual labour, who tended to reach a higher postoperative power level at six months in their injured elbow compared with their weaker unaffected non-dominant elbow.

An important finding is that drilling offered no advantage to the final outcome, but was, in fact, deleterious for a number of reasons: there was more postoperative pain (occasionally necessitating overnight admission), less postoperative elbow movement, more wound bleeding, more internal dissection was required to site the drill holes accurately, the postoperative pain took longer to resolve, and there was less pain difference improvement at three and six months on the Likud scale compared with the non-drilled elbow. These differences were especially apparent in patients who had bilateral Nirschl releases where one elbow was drilled and the other was not. The patients that benefitted most from the operation were those that rated their preoperative pain very high at the first consultation and were therefore really disabled by their condition.

CONCLUSION

The Nirschl technique for tennis elbow release is effective for resolving refractory lateral epicondylitis. However, these results suggest that drilling or decorticating the bone does not offer any benefit and should be avoided.

1 Nirschl RP, Pettrone FA. Tennis elbow. The surgical reatment of lateral epicondylitis. 7 Bone foint Surg [Am] $1979 ; 61: 832-9$.

2 Bankes MJ, Jessop JH. Day-case simple extensor origin release for tennis elbow. Arch Orthop Trauma Surg 998:117:250-1.

3 Newey MC, Patterson MH. Pain relief following tennis elbow release. $\mathcal{F}$ R Coll Surg Edinb 1994;39:60-1.

4 Russell IT. Clinical trials and evaluation of surgical Russell IT. Clinical trials and
procedures. Surgery $1984 ; 196-9$.

Take home message

Tennis elbow is a very common, disabling, and yet mismanaged condition. Early surgical treatment, when appropriate, is recommended. On the basis of this and other studies, Nirschl release but without the drilling component is recommended. 